\title{
AVATARES DE LA PRODUCCIÓN DE MEZCAL EN LA REGIÓN MIAHUATECA DE LA Sierra Sur de OAXaca
}

\section{Vicissitudes of Mezcal Production in the Miahuateca Region OF THE SOUTHERN HighLANDS OF OAXACA}

\author{
Joaquín Huitzilihuitl Camacho-Vera* \\ Juan Manuel Vargas-Canales** \\ Pedro Durán-Ferman***
}

DOI: http://dx.doi.org/10.29043/liminar.v19il.794

\begin{abstract}
Resumen: El mezcal, como todos los productos artesanales ligados al territorio, asume propiedades vinculadas con lo que se ha denominado terruño o terroir. La historia de la bebida, sus propiedades, sus procesos tecnológicos, sus sabores y sus usos son la historia misma del territorio y de las comunidades en las cuales se produce mezcal. En este trabajo se describen las condiciones del sistema de producción de mezcal en la región Sierra Sur del estado de Oaxaca, México, y se analizan las transformaciones generadas por el auge nacional e internacional de la demanda. Se encontró que, en la actualidad, el sistema productivo miahuateco está construido a partir de subsistemas territoriales que hacen uso de los recursos sociales, culturales y naturales de los distintos núcleos comunitarios de la región. En dichos subsistemas, las unidades de producción se manejan bajo una lógica de unidad de producción rural familiar campesina. Se piensa que el incremento, durante los últimos años, de los precios pagados al productor puede contribuir a la continuidad generacional de la producción artesanal.
\end{abstract}

Palabras clave: mezcal, denominación de origen, circuitos cortos de comercialización, producción familiarcampesina, producción artesanal.

Abstract: Mezcal, like all artisan products linked to a territory, takes on properties related to what has been termed terroir. The history of the drink, its properties, its technological processes, its flavors, and its uses are the history of the territory and the communities where mezcal is produced. This paper describes the mezcal production system in the Southern Highlands region of the state of Oaxaca, Mexico, and analyzes the transformations attributable to the domestic and international boom in demand. Currently, the Miahuateco productive system is based on territorial subsystems that make use of the social, cultural, and natural resources of the different communities in the region. In these subsystems, production units are managed by rural peasant families. Arguably, the increase in prices paid to the producer in recent years may help assure that artisanal production is continued from one generation to the next.

Keywords: mezcal, designation of origin, short trade channels, peasant family production, artisanal production.

\footnotetext{
* Joaquín Huitzilihuitl Camacho Vera. Doctor en Problemas Económico Agroindustriales por la Universidad Autónoma Chapingo, México. Profesor-investigador de la División de Estudios de Postgrado de la Universidad de la Sierra Sur, Oaxaca, México. Temas de especialización: socioeconomía, sustentabilidad, cambio tecnológico. Correo electrónico: camachove ra@yahoo.com.mx. ORCID: https://orcid.org/0000-0003-4284-1927

** Juan Manuel Vargas Canales. Doctor en Problemas Económico Agroindustriales por la Universidad Autónoma Chapingo, México. Profesor de tiempo completo de la Universidad de Guanajuato, México. Temas de especialización: innovación tecnológica, cambio tecnológico,
}

especialización y competitividad. Correo electrónico: jm.vargas@ugto. mx. ORCID: https://orcid.org/0000-0003-1918-9395

*** Pedro Durán Ferman. Doctor en Problemas Económico Agroindustriales por la Universidad Autónoma Chapingo, México. Profesor-investigador del Instituto de Estudios Municipales de la Universidad de la Sierra Sur, Oaxaca, México. Temas de especialización: empresas rurales, sustentabilidad. Correo electrónico: pduranferman69@hotmail.com. ORCID: https:// orcid.org/0000-0002-8226-0513

Enviado a dictamen: 3 de marzo de 2020.

Aprobación: 25 de agosto de 2020. 


\section{Introducción}

E n la discusión de la modernidad alimentaria, los productos del terruño forman parte de un constructo actual que hace referencia a alimentos o bebidas que provienen de sistemas altamente localizados, en el sentido de que el territorio incorpora en ellos una enorme carga de especificidades que los hace prácticamente imposibles de imitar o producir en otro espacio geográfico (Duhart, 201l; Linck, Barragán y Casabianca, 2006; Muchnik, 2006). En este sentido, las condiciones particulares del ambiente y de los ecosistemas que los sustentan, así como los elementos culturales de la sociedad que los elabora, se incorporan todos como elementos constitutivos, tanto materiales como inmateriales, de tal manera que los dotan de propiedades organolépticas y simbólicas reconocibles; es decir, de genuinidad (Villegas y Cervantes, 2011).

Los productos de este tipo han sido tomados como motores de procesos de desarrollo, y a partir de ellos se han tratado de construir estrategias de acción y políticas públicas con fines muy ambiciosos, que van desde el aumento del ingreso en los hogares hasta la mejora de la calidad de vida de regiones completas. Como ejemplo de lo afirmado, los modelos de análisis e intervención territorial como los sistemas agroalimentarios localizados (Muchnik y Sautier, 1998; Requier, 1997), los sistemas productivos locales (Courlet y Pecqueur, 1992) e incluso los distritos industriales (Becattinni, 1979) han considerado los productos localizados como ejes de sus estrategias de intervención de los espacios rurales, tanto en Europa como en América Latina (Camacho et al., 2019).

Sin embargo, aun con sus enormes particularidades simbólicas y vínculos con el territorio y la cultura de la sociedad que los genera, los productos del terruño quedan atrapados dentro de la dinámica de la modernidad alimentaria regida por los circuitos capitalistas mundializados que forman parte del sistema agroalimentario global (Hernández y Villaseñor, 2014). Conforme los sistemas artesanales locales se conectan de manera más intensa con los ciclos capitalistas, se van convirtiendo en sistemas funcionales a su lógica de monopolio y de gran empresa, con lo que se ve erosionada su capacidad de dispersión de ingresos en los territorios y, por lo tanto, su capacidad para mejorar, en lo económico, la vida de las comunidades que los producen.

El mezcal, como todos los productos artesanales ligados al territorio, asume propiedades vinculadas con lo que se ha denominado terruño o terroir. En tal sentido, esta bebida no es algo inerte sino un actante (Latour, 2001) socialmente construido, con propiedades particulares derivadas de la coevolución entre la sociedad que lo ha elaborado y los ecosistemas y agroecosistemas que sustentan su producción. Así pues, la historia de la bebida, sus propiedades, sus procesos tecnológicos, sus sabores y usos son parte de la trayectoria misma del territorio y de las comunidades en las cuales se produce mezcal.

La región miahuateca y la producción de mezcal tienen un fuerte vínculo histórico y cultural que imprime particularidades y especificidades a esta bebida, emblemática del estado de Oaxaca, pero que también se identifica con otros territorios dentro de una macrorregión definida a partir de criterios normativos que construyen la Denominación de Origen Protegida Mezcal (DOP Mezcal). Podemos entonces considerar que el mezcal reúne todas las características para ser considerado un producto del terruño, y como tal tiene potencial para constituirse en eje de estrategias de desarrollo rural. Sin embargo, para que esto sea posible es necesario entender el sistema mezcalero con mayor profundidad que la sola cuestión tecnológica; esto es, conocer también sus entramados sociales y su inserción y funcionalidad para los mercados globales de bebidas alcohólicas.

De acuerdo con la definición contenida en la Norma Oficial Mexicana 070 (NOM 070) (Comité Consultivo Nacional..., 2016), el mezcal es una bebida alcohólica que se obtiene por destilación de jugos derivados de la fermentación de cabezas cocidas de maguey de cualquier especie de agave, siempre y cuando sea cosechado dentro del área considerada como protegida por la DOP Mezcal (Comité Consultivo Nacional..., 2016). Sin embargo, esta definición formal no hace justicia a la enorme riqueza en cuanto a la elaboración de mezcales 
que se han desarrollado en distintas regiones del país, pues algunas de ellas actualmente se encuentran fuera del territorio delimitado por la DOP Mezcal. Como lo señalan Plascencia y Peralta (2018), los mezcales conforman un grupo amplio y heterogéneo de bebidas destiladas que hacen uso del agave como materia primordial para su proceso. A dicha heterogeneidad, además de la diversidad biológica de los agaves, abonan la enorme diversidad cultural de los espacios en donde se produce y, por tanto, la enorme pluralidad de prácticas culturales relacionadas con su producción y consumo.

En los últimos años, el mezcal se ha convertido en una de las bebidas de moda en el mercado mundial de bebidas alcohólicas. Su enorme aceptación está relacionada con cierta carga de exotismo y apreciación de genuinidad y artesanalidad que le da particularidades a su producción y comercialización y lo diferencia de otros sistemas de bebidas similares como el tequila. No obstante, los enormes intereses económicos, tanto nacionales como transnacionales, han asumido el control de su producción y comercialización y de las instituciones que las regulan, con lo cual excluyen y soslayan a productores y territorios de los potenciales beneficios del comercio de esta bebida (Flores et al., 2018; Renard y Domínguez, 2019).

En este trabajo se describen, de manera general, las condiciones del sistema de producción de mezcal en la región Sierra Sur del estado de Oaxaca, uno de los territorios que forma parte de la región protegida por la DOP Mezcal. Se analizan las transformaciones generadas por el auge nacional e internacional de la demanda de mezcal genuino, y se discurren algunas consideraciones sobre las posibilidades que tienen los productores artesanales de la región de beneficiarse de la prosperidad que ha tenido la bebida en estos últimos años y sobre las repercusiones de la reconfiguración de los mercados internacionales sobre este territorio en particular.

El abordaje teórico de este trabajo se realiza, en parte, desde la antropología de la alimentación, en cuanto a la propiedad de localización de los productos alimentarios reconocidos como tradicionales o típicos. Desde este enfoque, un alimento localizado puede considerarse como tal si en su elaboración se incorporan, además de los rasgos propios que la geografía da a sus materias primas, cualidades - tangibles o intangibles - particulares de la cultura local. Esta perspectiva es retomada por la teoría del desarrollo para explicar la organización de los espacios y la prosperidad económica de ciertos territorios, a partir de la constitución de sistemas agroalimentarios localizados (SIAL) sustentados por una red de pequeñas y medianas agroindustrias rurales. Un SIAL explica la competitividad de ciertos sistemas alimentarios a partir de la obligada incorporación de los rasgos socioculturales como principal fuente de competitividad territorial. Se asume también una perspectiva analítica bajo la idea de que las unidades de producción son los elementos básicos del complejo sistema productivo y de que su evolución en el territorio ha estado a merced de la construcción de una nueva realidad en el ámbito rural, a partir del avance de la modernidad y de los fenómenos relacionados con su conexión con los procesos de globalización. Es decir, la nueva ruralidad forma parte del marco explicativo desde el cual se interpretan los hechos sociales descritos en relación con la producción y comercialización de mezcal en este territorio oaxaqueño.

\section{Metodología}

El área de estudio, el distrito de Miahuatlán, está constituida por 32 municipios que conforman una de las principales regiones mezcaleras incluidas dentro de la DOP mezcal: la Sierra Sur de Oaxaca. Además de Miahuatlán, la región Sierra Sur está conformada por los distritos de Sola de Vega y Yautepec. Los tres distritos comparten una importancia similar en cuanto al volumen de producción de mezcal, número de mezcaleros y superficie cultivada de maguey (COPLADE, 2016).

Este trabajo se desarrolló a partir de una revisión documental y entrevistas, tanto enfocadas como a profundidad, con productores de la región de estudio y otros actores relacionados de manera directa o indirecta con el sistema de producción y comercialización de mezcal. En cuanto a la revisión documental, se utilizaron artículos y ensayos científicos que hacían énfasis en la realidad 
social alrededor de la producción de mezcal. Aunado a lo anterior se utilizaron documentos de diagnóstico del Consejo Regulador del Mezcal y de instituciones de planeación del estado de Oaxaca. Las entrevistas se realizaron durante 2019, para lo cual se seleccionó a productores de mezcal mediante una técnica de muestreo no probabilístico, por conveniencia, a través de la identificación y referencia directa de otros productores. Este tipo de muestreos en red es de especial utilidad cuando se desconoce el universo de elementos que constituyen el espacio muestral.

Las entrevistas a profundidad se ajustan a un modelo de conversación entre iguales orientada a comprender las perspectivas que tienen los informantes respecto a sus vidas y sus experiencias expresadas en sus propias palabras (Robles, 2011). La entrevista a profundidad se constituye a partir de "encuentros reiterados cara a cara entre el investigador y los informantes" (Taylor y Bogdan, 1990). En esta investigación, se realizaron distintos encuentros cara a cara con productores de mezcal de comunidades del distrito de Miahuatlán de Porfirio Díaz.

Por su parte, las entrevistas focalizadas (focused interview) (Merton, 1946; Merton, Fiske y Kendall, 1990) se corresponden con instrumentos semiestructurados que tratan un tema específico. Para este trabajo, el guion de este tipo de instrumentos obedeció a una serie de dimensiones que pretendieron reconocer los elementos históricos de la actividad mezcalera en cuanto a la producción, comercialización y usos tecnológicos, así como a los cambios ocurridos en la actividad productiva. Además se delimitaron las perspectivas y las opiniones respecto a la situación actual del sistema y la relación con los organismos institucionales. En general, las entrevistas se concentraron en las principales comunidades productoras: San Luis Amantlán, Miahuatlán de Porfirio Díaz, Sitio Xitlapehua, Mengolí y Yegachín.

Los relatos obtenidos se organizaron a fin de obtener una perspectiva detallada sobre la evolución de la estructura del sistema de producción de mezcal en cuanto a sus elementos componentes y su modificación en el tiempo - en particular sobre los actores dedicados a la elaboración de la bebida y los intermediarios-. En ese mismo sentido, se buscó construir un relato en el tiempo sobre la tecnología utilizada y los factores fundamentales que explican su modificación. Finalmente, con la trayectoria del sistema como contexto, y en conjunción con información nacional e internacional, se construyeron escenarios posibles y recomendaciones plausibles para mejorar el impacto del sistema productivo sobre la calidad de vida de los hogares involucrados con la actividad mezcalera.

\section{El mezcal, orígenes y actualidad: historia y contexto nacional e internacional}

La producción de los destilados de agave puede considerarse como tradicional de varias regiones de la República mexicana y con arraigo de varios cientos de años de antigüedad. El origen del mezcal es incierto, pero su proceso de elaboración es compartido por varias bebidas destiladas ancestrales de distintas regiones del país, como el sotol, la raicilla, la bacanora y el tequila mismo, entre otras a las que se podría llamar genéricamente mezcales (Salazar, 2007; Zizumbo y Colunga, 2007). Recientes estudios arqueológicos hacen suponer que las bebidas de este tipo son de origen prehispánico (Goguitchaichvili et al., 2018), lo que contradice las hipótesis de mayor popularidad hasta el momento, por las cuales se suponía que la tecnología para realizar el proceso de destilación solo fue conocida en México hasta después de la llegada de los españoles, como una tecnología importada en los periodos posteriores a la conquista (Walton, 1977).

En un estudio realizado en el centro ceremonial de Cacaxtla, en el estado de Tlaxcala, Goguitchaichvili et al. (2018) encontraron evidencia de infraestructura para horneado de maguey que fue utilizada en periodos tan tempranos como de 878 a 693 a. C. Hasta antes de estos trabajos, preponderaba la idea del origen colonial de la elaboración del mezcal, como producto de la transferencia de tecnología de destilación del viejo mundo hacia las costas mexicanas, en concreto a las costas del Pacífico. De acuerdo con Valenzuela, Regalado y Mizoguchi (2008), la tecnología de destilación pudo llegar a la Nueva España por varias vías, pero la utilizada 
en la obtención de mezcales muestra evidencias de una relación más directa con la tecnología filipina para la obtención de destilados derivados de la fermentación de la planta de coco.

El uso de destiladores de tipo filipino estuvo directamente relacionado con la obtención de bebidas alcohólicas derivadas de la fermentación de los brotes de la palma de coco, y de ahí se intuía que habría pasado a la producción de mezcal, una vez que las bebidas del primer tipo fueron prohibidas en la primera década del siglo XVII (Zizumbo y Colunga, 2008). Sin embargo, Goguitchaichvili et al. (2018) han reunido evidencia que dirige la discusión hacia el posible origen prehispánico de la bebida, lo que implicaría un desarrollo tecnológico mesoamericano propio en cuanto a los procesos de destilación. Los descubrimientos relacionados con la destilación prehispánica modifican la ruta del conocimiento y punto de origen posible del mezcal. Con las evidencias anteriores se pensaba que las costas de Jalisco eran el punto de origen y dispersión de la tecnología de destilación y, por lo tanto, punto de origen de la elaboración de mezcales. Sin embargo, si la tecnología fue generada en el periodo prehispánico, es lógico que se haya desarrollado en el centro de Mesoamérica y que de ahí se haya difundido hacia sus periferias. No obstante, esta última es una hipótesis aún en construcción.

\section{El mezcal en la Sierra Sur: historia, persecución, resistencia y cambio}

De la producción de mezcal existen reportes desde mediados del siglo XVI, aunque la primera referencia como mezcal data de principios del siglo XVII (Lozano, 2015). Por esos años la producción de vinos y ciertos licores fue prohibida en la Nueva España con el argumento de que ocasionaba daños a la salud de la población, pero la prohibición en realidad tenía como fin proteger las importaciones de licores que se hacían desde la metrópoli (Walton, 1977).

Para los siglos XVII y XVIII, la elaboración del mezcal se vinculó con grandes haciendas propiedad de españoles, ubicadas en Nueva Galicia y Nueva Vizcaya, que surtían con la bebida a las zonas mineras de esas mismas regiones (Lozano, 2015; Walton, 1977). A principios del siglo XIX se eliminó la prohibición a la producción y comercialización de mezcales a través de un bando firmado por el virrey de la Nueva España (Bazán, 1964). A principios de siglo XX, durante el periodo revolucionario y posrevolucionario, la producción de mezcal se vio sujeta a fuertes presiones impositivas que disminuyeron la rentabilidad de la producción (Anaya, 2018; Iwadare, 2015).

En Oaxaca, el mezcal ha estado ligado desde el periodo colonial e independiente a la pequeña producción y al comercio en pequeña escala de campesinos indígenas. Entre las mercancías denominadas "efectos de indios", se reconoce que el comercio de vino mezcal representaba una parte importante del volumen comerciado por la población indígena (Sánchez, 1999). No obstante, como en otras regiones del país, buena parte del auge en la producción de la bebida se produjo durante la expansión capitalista del sistema hacendario, como parte de los productos que se comercializaban en los centros urbanos.

En el distrito de Miahuatlán, de la región Sierra Sur, al igual que en otras regiones de Oaxaca, si no es que en todo su territorio, la producción y el consumo de mezcal han estado inherentemente ligados a la vida de las comunidades como parte de la cultura de la fiesta y del trabajo, aun antes de su notable éxito en el mercado de bebidas alcohólicas. En cada comunidad, por pequeña que fuera, se podían encontrar uno o dos "palenqueros" que surtían el volumen necesario para la comunidad y para la venta e intercambio en los mercados locales.

En la región miahuateca, el mezcal ha formado parte habitual de la vida rural agrícola, como un elemento básico para el consumo durante o después de las extenuantes jornadas de trabajo. Se bebía esta "esencia fuerte" (ma nguiéedz, en una de las variantes de zapoteco de la región) para recuperar el aliento durante las caminatas y el trabajo diario y, al final de la jornada, para quitar el cansancio extremo y estar listo para el siguiente día. Sin embargo, durante las últimas décadas los espacios rurales han sido sujetos de profundas transformaciones, y con ellas la vida campesina, marcada en sus ritmos por los ciclos agrícolas, se vio trastocada por la necesidad de 
mantener la reproducción social de los hogares rurales. La pluriactividad (De Grammont, 2004), en la que siempre se había concedido mayor peso a la agricultura, se fue decantando hacia el trabajo asalariado - agrícola y no agrícola-y el comercio, y con ello, en algunos casos disminuyó la producción de mezcal; incluso, en algunas comunidades de la región desapareció completamente $\mathrm{y}$, por ende, la cultura asociada a ella.

La disminución de la importancia relativa de la agricultura campesina como medio para la reproducción social de los hogares rurales implicó la disminución de superficies destinadas a cultivo tradicional para consumo e intercambio campesino. En cierta medida, este abandono posibilitó la recuperación del medio natural, pero también se abandonaron los caminos cosecheros que posibilitaban el acceso a esos espacios. Lo anterior implica que si bien en la región, en la actualidad, existe una cantidad considerable de material silvestre para sustentar la producción de la bebida, el acceso a esos recursos es más difícil. En ese mismo sentido, variedades de maguey de enorme tradición como los cuixes (Agave karwinskii), que eran utilizados como linderos, disminuyeron en abundancia.

La historia del mezcal en la región, al igual que en la mayor parte de las entidades del país, había sido una historia de clandestinidad y persecución. Solo hasta recientes décadas, de la mano del auge de la bebida, ha dejado de tener carácter ilegal, hasta cierto punto. Buena parte de los productores de mayor edad recuerdan aún los predicamentos que tuvieron que superar las anteriores generaciones a fin de mantener la producción de mezcal como una actividad económica factible. Tanto la cultura como la tecnología respondían directamente a esta situación de riesgo continuo.

Por lo regular, los palenques para el procesamiento de la bebida se encontraban situados en puntos de difícil acceso, cerca de una fuente de agua de carácter permanente o estacional, en barrancas en medio de los cerros o en las riberas de los ríos y arroyos alejados de los caminos habituales. Las instalaciones tenían un carácter temporal, sin edificaciones ni equipos difíciles de mover. El piso para la molienda del maguey cocido estaba fabricado con tierra apisonada bajo un coberti- zo de materiales obtenidos del entorno. El proceso de molienda se realizaba a mano, con el uso de mazos de madera de fabricación propia a partir de los árboles de maderas duras de la región. Las barricas y ollas para el almacenamiento del mosto y del producto final y el equipo de cobre para la destilación representaban la principal inversión, de ahí que su pérdida, decomiso, hurto o destrucción significaran un descalabro económico significativo para el productor mezcalero.

De acuerdo con los relatos de los artesanos, había un hostigamiento constante por parte de distintas autoridades, principalmente del ámbito estatal, relacionadas con el cobro de impuestos y la regulación comercial. Cuando un palenque era descubierto, era habitual que se produjera un embargo de los alambiques o, en casos extremos, la destrucción del equipo y de la bebida almacenada y la detención del productor o trabajador que hubiera sido encontrado en el lugar. La comercialización y el traslado del producto a los mercados regionales tenían el mismo carácter furtivo que la producción. Los productores o intermediarios transportaban el producto en cántaros a lomo de burro, desde sus comunidades de origen hasta el principal centro de consumo: la ciudad de Miahuatlán. El recorrido se realizaba por la noche o la madrugada, a fin de evitar cualquier inconveniente con algún tipo de autoridad. Al llegar a la zona urbana, se hacían las entregas en la vivienda misma de los comerciantes al detalle, con quines se tenía ya un acuerdo previo.

En las historias de los mezcaleros, al hacer remembranza de las vicisitudes de las generaciones que les antecedieron, es frecuente que se relate, desde una perspectiva épica, la persecución de la que era objeto el productor de la bebida. En esas narraciones el rol negativo se asigna siempre a la figura del representante de gobierno que llegaba a las comunidades con la intención expresa de buscar e imponer sanciones a las personas que se dedicaban a esta actividad sin reportar los impuestos correspondientes. Si bien en la actualidad tal persecución ya no ocurre, hay una narrativa similar por parte de los organismos reguladores y los actores de mayor poder dentro del sistema, que busca desvirtuar la pequeña producción que no se ajusta a 
las condiciones marcadas por la normativa de la denominación de origen. De esta forma, territorios fuera de los límites marcados por la DOP Mezcal, con una enorme tradición mezcalera, se encuentran excluidos de los mercados formales de la bebida. Más aún, en los mismos territorios que comprenden la denominación de origen, los productores no certificados pueden ser también excluidos.

\section{Actualidad del sistema productivo y su adaptación a la dinámica internacional}

En la actualidad, el sistema productivo miahuateco está construido a partir de subsistemas territoriales centrados en lo productivo y en los recursos sociales, culturales y naturales de los distintos núcleos comunitarios de la región. Como ya se ha comentado, en la mayoría de las poblaciones del distrito se elaboraba la bebida de manera tradicional, con variantes en cuanto a los recursos biológicos que sustentan la actividad, pero con una relativa homogeneidad tecnológica y cultural. Sin embargo, los enormes cambios sociales ocurridos en la ruralidad mexicana durante las últimas décadas han erosionado la actividad y, en algunos casos, ha desaparecido de la vida comunitaria de las localidades - como en la región de los Coatlanes, de la misma Sierra Sur-. En donde ha permanecido lo ha podido hacer por su interacción con mercados locales y con mercados urbanos como el de la ciudad de Miahuatlán y de otras ciudades aledañas, y aun de la misma capital del estado.

Buena parte de la transformación tecnológica de las unidades de producción está relacionada con la promoción de los programas de desarrollo, impulsados desde los ámbitos federal y estatal, que se han dirigido básicamente al cambio tecnológico, con miras a la mejora de la eficiencia productiva y a la promoción de la formalización de la actividad y el apego a la normativa creada alrededor de la denominación de origen. Así pues, los programas de fomento económico de la actividad han promovido el uso de molinos de piedra o eléctricos, pisos de concreto, techados, división por áreas de operaciones y uso de instrumentos de monitoreo como alcoholímetros. En términos llanos, las instituciones del
Estado hacen esfuerzos por modernizar la producción de mezcal aun cuando esa modernización tecnológica puede alterar la lógica de unidad familiar y retroactuar, de manera negativa, sobre la carga simbólica valorada en este tipo de bebida.

Desde mediados de los ochenta a la fecha, en lo que constituye el llamado boom mezcalero (Borrego y Becerril, 2018), el mercado nacional e internacional de la bebida no ha dejado de crecer. En ese mismo periodo se ha construido una institucionalidad que busca favorecer a los actores de mayor poder representados por las envasadoras nacionales e internacionales. Así pues, las envasadoras tratan de que los organismos certificadores, reguladores y la normativa en general favorezcan la acumulación de capital en los circuitos de comercialización nacional y, en fechas más recientes, en los de índole internacional. Esta presión hacia el cambio se transmite al sistema productivo local mezcalero como una constante incitación al cambio tecnológico por parte de las dependencias de gobierno relacionadas con el sector, bajo el argumento de una mayor eficiencia tecnológica y un mayor potencial de rentabilidad económica.

En la región miahuateca, como en la mayoría de las regiones mezcaleras de la entidad y del país, el predominio de la perspectiva industrial de eficiencia económica se ha traducido también en una presión sobre los componentes social y natural del sistema mezcalero. En cuanto al componente natural, la sustracción de semillas, hijuelos y especímenes adultos de las distintas especies de magueyes mezcaleros ha constituido un proceso constante de erosión de los sistemas biológicos naturales. En recientes fechas, se ha dado una enorme presión sobre las distintas especies de magueyes silvestres de la mano de la necesidad de aumentar la materia prima disponible, sobre todo para los procesos más industriales. Cabría hacer aquí una acotación en cuanto a la consideración de mezcal industrial, definición relacionada de acuerdo con la norma con especificidades del proceso, pero que en realidad debería obedecer más a criterios de volumen de producción y características de búsqueda de eficiencia tecnológica y financiera a fin de maximizar la rentabilidad económica. En este sentido, 
las unidades industriales más intensivas necesitan una provisión constante de materias primas que derivan de los sistemas agrícolas, también intensivos, o de la sobreexplotación de los sistemas naturales.

En contraposición, las unidades artesanales funcionan más bajo la lógica de unidades familiares pluriactivas, aunque, en los años recientes, con mayor inclinación hacia la especialización en la manufactura de mezcal. Aun así, la producción artesanal tiene un alto componente de agricultura campesina típico de una unidad de producción rural. Si bien, al igual que los sistemas industriales, se ha comenzado a hacer un mayor uso de los agaves silvestres por la dinámica de los mercados, la intensidad de la explotación es mucho menor. Más aún, buena parte de los mezcaleros están adaptando ya para el cultivo las especies silvestres de mayor interés comercial —como tepeztate y tobalá, entre otras-. Aunque ya existen en la región superficies con monocultivo de maguey, lo más frecuente es encontrarlo imbricado con maíz y frijol, es decir, como parte aún de sistemas campesinos.

En síntesis, la actividad de los palenques artesanales regionales se ajusta, entre otras variables, a las necesidades económicas de los hogares. En ese sentido, es comprensible que los volúmenes de producción de mezcal sean moderados y los periodos de trabajo discontinuos en el tiempo. Las actividades campesinas y las condiciones climáticas son aún condicionantes del tiempo dedicado a la producción de la bebida, es decir, los ciclos productivos del mezcal se sujetan a las condiciones climáticas y a los ciclos agrícolas. En este sentido, lo que pareciera actuar en contra de la rentabilidad de la actividad o del potencial de retorno económico favorece el cuidado sobre la calidad final del producto. En otras situaciones, y bajo una premisa de maximización de utilidades, los mezcaleros podrían, por ejemplo, hacer uso de plantas de agave que aún no han llegado a la madurez, como ocurre en otras regiones de Oaxaca más vinculadas con la producción industrial.

En cuanto a las presiones de la institucionalidad sobre el componente social, estas se habían traducido en una presión sobre el precio pagado al productor. Tal como sucede con otros sistemas agrícolas y agro- industriales, hay un eslabón que tiene una posición de dominio sobre los demás elementos relacionado con el control sobre la producción o comercialización. En el caso del mezcal, son las empresas envasadoras de mayor tamaño y con mejores vínculos con los mercados nacionales e internacionales las que ejercen ese dominio en los distintos territorios, tanto fuera como dentro de la DOP. No era diferente para el caso específico del mezcal miahuateco, con el que, bajo el argumento de que la producción de la bebida no cumple con la certificación del producto, las empresas envasadoras negociaban acuerdos ventajosos que se traducían en precios bajos a productores y bajas rentabilidades de la actividad (COPLADE, 2016).

Sin embargo, a partir del año 2015 la situación del precio pagado al productor se ha modificado. Los incrementos sustanciales en la demanda nacional e internacional han presionado a la alza los precios de la bebida, lo que ha terminado por impactar en los precios pagados al productor. ¿Qué factores han permitido que se dé esta transmisión? Básicamente, que la misma DOP limita el crecimiento drástico de la oferta nacional y, en el ámbito de la Sierra Sur, la existencia y el fortalecimiento de canales cortos de comercialización. Se puede afirmar entonces que el incremento del precio es producto de varias circunstancias que se originan, en su mayoría, fuera del sistema productivo local miahuateco y que en conjunto producen este efecto. El auge de la bebida favorece que la demanda siga creciendo, mientras que los límites geográficos establecidos por la DOP no permiten incrementos abruptos de la oferta — a pesar de las presiones por ampliar la DOP-. A estas dos circunstancias se añade la peculiaridad de la preferencia del mercado por productos vinculados con el territorio, y que este vínculo sea evidente en lo organoléptico y lo simbólico.

En definitiva, el incremento de los precios de los últimos años ha beneficiado de manera significativa a los pequeños productores miahuatecos. Sin embargo, esta mejora en sus condiciones no se ha dado en virtud de una mayor participación del productor como actor de la conducción del sistema, sino más bien por la transmisión de los efectos del incremento constante 
de la demanda local, nacional e internacional. En este sentido, el productor se ha convertido en un tomador de precios que trata de adaptar su sistema de producción a la demanda de mezcales de alto valor económico, elaborados a partir de especies específicas de agave.

El grueso de los productores no se ha adherido a la certificación como recurso garante de la comercialización exitosa de su producto. Los productores se mantienen alejados de la idea de certificación promovida desde los organismos institucionales, en buena parte porque la obtención de la certificación imposibilita la comercialización bajo su sistema actual de oportunidad de menudeo y de canales cortos. En este sentido, los productores consideran que si certificaran su producción tendrían poca oportunidad de comercializar a pie de palenque, como lo han hecho de manera habitual. Más aún, en virtud del aumento exponencial de los precios, estos mismos circuitos cortos de comercialización les permiten obtener precios que no difieren significativamente de los del mezcal certificado. Por esta situación, incluso los productores que cuentan con certificación prefieren vender la mayor parte de su producción sin este distintivo.

Aun cuando las unidades campesinas permanecen como el corazón del sistema mezcalero de la región, la enorme influencia de la demanda exterior al territorio ha contribuido a la modificación de la esencia del producto. Es decir, la bebida que se conoce ahora como mezcal, conforme avanza el tiempo, va perdiendo los rasgos de lo que las generaciones anteriores consideraron como tal. Cuando se analizan los cambios en el consumo local, los pobladores afirman que "la gente de antes", "los mayores", eran buenos para tomar el mezcal, con lo que hacen referencia tanto a la cantidad consumida como a la frecuencia y al contenido alcohólico de la bebida. Tanto los pobladores en general como los artesanos mezcaleros coinciden en que el mezcal que tomaban en décadas anteriores era una bebida más fuerte y, en cierto sentido, más auténtica en tanto que respondía a una realidad de mayor trabajo físico. Esto es claro si se considera que parte de los procesos y, por tanto, el producto final, se han tenido que amoldar a los gustos de las poblaciones urbanas. En este sentido, se prefieren ahora mezcales con un menor contenido alcohólico y con notas organolépticas que denoten el tipo de maguey del que se obtuvo. Ya no interesa, pues, que la bebida "dé fortaleza”, sino que sea lo más agradable posible al gusto. Es claro que esa tendencia es la que se va a generalizar y la que dominará los ámbitos de la producción.

En este sentido, autores como Bautista, Orozco y Terán (2015) afirman que en Oaxaca la producción artesanal de mezcal está en franco declive, en especial en las regiones de mayor fama y reconocimiento como Matatlán. En buena parte, este declive ha sido ocasionado por las propias políticas institucionales de fomento de la bebida, orientadas hacia la promoción de los modelos centrados en procesos industriales que la alejan de su carácter artesanal. Esta situación no parece ser la predominante en la región miahuateca, en donde aún se conservan la mayor parte de las características artesanales del proceso, tanto en su componente tecnológico como en cuanto a su condición de pequeña escala y trabajo vivo (Camacho et al., 2019); sin embargo, las presiones de la demanda ya se hacen presentes.

\section{El futuro del mezcal miahuateco: exclusión frente a reapropiación}

¿Cómo se ve a futuro la situación del mezcal miahuateco? En lo general, los productores mezcaleros miahuatecos pueden considerarse como actores relativamente pasivos del sistema que reaccionan a las condiciones del mercado. Tradicionalmente, como en la mayoría de las actividades agrícolas rurales, existe un bajo nivel de asociación y preferencia a conducirse como entes individuales. Esto es entendible si se considera que las unidades productivas funcionan bajo lógicas familiares de producción, y no bajo criterios de eficiencia empresarial.

Sin embargo, para los intereses de los grupos económicos que dominan el sistema mezcal en el país, la moderación propia de la lógica económica campesinaartesanal no es funcional a las expectativas de rentabilidad del comercio de la bebida, por lo que ese tipo de lógica buscará limitarse, eliminarse o restarle poder, aun cuando desde el discurso, el simbolismo y la estética se 
argumente en sentido contrario. Es por esta razón que existen enormes presiones para ampliar la DOP Mezcal hacia otros estados de la República, lo que implica una confrontación entre los intereses empresariales que dominan el sistema mezcal en la actualidad y los intereses económicos que pretenden incursionar en la actividad dada su enorme rentabilidad y su crecimiento continuo. $\mathrm{Y}$, aunque el argumento sea la necesidad de incluir productores tradicionales excluidos, en la realidad lo que se pretende es integrar territorios que puedan proveer materia prima - ya sea silvestre o cultivada - y la posibilidad de la denominación mezcal a los destilados que ahí se produzcan, aunque no haya ningún componente simbólico que integrar en la bebida.

Durante los próximos años es seguro que en la región aumentará la superficie sembrada con agaves silvestres, y con ello la producción de mezcales de alto valor comercial. Los productores artesanales iniciaron sus propios cultivos en cuanto se percataron de la enorme diferencia de precios al que se puede vender al consumidor final, como una forma de prever sus necesidades de abasto futuras. Dado el incremento de la rentabilidad de la producción de mezcal, el sistema artesanal tiene posibilidades de continuar bajo la lógica familiar y de mantenerse en el tiempo como una actividad de sustento. La continuidad generacional será posible siempre y cuando los precios recibidos por el productor mantengan la tendencia actual.

Sin embargo, es muy factible que aumenten en cantidad las unidades de producción con lógicas más industriales. Las nuevas unidades de producción se alejarán de las lógicas familiares y se sujetarán más a criterios de maximización de utilidades bajo una administración más empresarial y con menores vínculos con los mercados locales. Estas unidades buscarán aprovechar la DOP para satisfacer las demandas nacionales e internacionales y competirán con el sistema artesanal por materia prima e incluso por mercados.

\section{Conclusiones}

El sistema productivo de mezcal en Miahuatlán se corresponde con un sistema artesanal cuyas unidades de producción se manejan bajo una lógica de unidad de producción rural familiar campesina. Está conformado por una estructura de pequeños y medianos productores que ofrecen su producción a través de canales cortos de comercialización lo que, de manera muy frecuente, significa vender a pie de palenque.

El sistema productivo tiene una enorme raigambre territorial y está vinculado a la historia de las distintas comunidades en donde se practica la actividad. A lo largo de los años, principalmente a partir del boom mezcalero, se han dado una serie de transformaciones tecnológicas y en la estructura del sistema que no han minado la naturaleza artesanal de los procesos ni la lógica productiva centrada en las necesidades familiares. Sin embargo, sí se han modificado algunas de las características organolépticas de la bebida, como una forma de adaptarse a las nuevas demandas de los mercados y a las condiciones impuestas por las regulaciones de los distintos organismos que coordinan el sistema nacional.

El incremento de los precios pagados al productor de los últimos años contribuye a la continuidad generacional de la producción artesanal. Sin embargo, es importante que se promueva la construcción de canales cortos de comercialización, de tal manera que se ponga en contacto a los productores locales con mercados nacionales y transnacionales que aprecien las cualidades de los mezcales artesanales no solo en lo referente a aspectos de calidad organoléptica, sino de la valoración de lo simbólico y la ética de la producción de pequeña escala.

Como recomendación, sería importante que los actores del sistema artesanal regional aprovechen la bonanza de precios y consoliden y multipliquen sus canales cortos de comercialización. En ese mismo sentido, es fundamental que se trabaje para promover la cualidad artesanal como la principal característica distintiva de la bebida, no exclusivamente desde los argumentos de sabor y aromas como se hace en la actualidad, sino desde la esencia verdadera de lo artesanal en el campo simbólico de la tradición, la genuinidad y la autenticidad. Para una empresa industrial es fácil imitar aromas y sabores, y con ellos suplantar las bebidas tradicionales; sin embargo, si se conduce la promoción 
de la valoración hacia la rusticidad y la tradición, los productores artesanales pueden tener una mayor posibilidad de tomar el control del sistema mezcal. Estas acciones son poco efectivas si se emprenden desde las unidades individuales; sin embargo, la construcción de una o más marcas colectivas da la posibilidad de consensuar una normatividad que garantice la c(u)alidad artesanal. Más aún, la redefinición de lo artesanal y su promoción como estrategia de posicionamiento en el mercado pueden también constituirse en una estrategia de defensa y revaloración de una lógica de producción campesina $y$, con ello, de una forma de vida que, ante la crisis civilizatoria (Bartra, 2008) actual, se perfila como la única vía factible.

\section{Referencias}

Anaya Merchant, Luis (2018). "Producto de alcohol en el México del porfiriato”. En Inventio. La Génesis de la Cultura Universitaria en Morelos, 3(6), 27-34.

Bartra, Armando (2008). "Fin de fiesta: el fantasma del hambre recorre el mundo". En Argumentos, 21(57), 15-31.

Bautista, Juan Antonio, Sergio Orozco Cirilo y Edit Melchor Terán (2015). "La disminución de la producción artesanal de mezcal en la Región del mezcal de Oaxaca, México". En Revista Mexicana de Ciencias Agrícolas, 6(6), 1291-1305.

Bazán Alarcón, Alicia (1964). "El Real Tribunal de la Acordada y la delincuencia en la Nueva España". En Historia Mexicana, 13(3), 317-345.

Becattini, Giacomo (1979) "Dal 'settore' in-dustriale al 'distretto' industriale. Alcune considerazioni sull'unità di indagine dell'economia indus-triale". En Revista di Economia e Politica Industriale, (1), 35-48.

Borrego Rivera, Georgina y Javier Becerril Isidro (2018) "Estrategias comerciales para el incremento de ventas de mezcal en el extranjero". En Hospitalidad-ESDAI, (33), 95-118.

Camacho Vera, Joaquín Huitzilihuitl, Fernando Cervantes Escoto, Alfredo Cesín Vargas y María Isabel Palacios Rangel (2019). "Los alimentos artesanales y la modernidad alimentaria". En Estudios Sociales. Revista de Alimentación Contemporánea y Desarrollo Regional, 29(53).
Comité Consultivo Nacional de Normalización de la Secretaría de Economía (2016). Norma Oficial Mexicana NOM-070-SCFI-2016, Bebidas AlcohólicasMezcal-Especificaciones. México.

COPLADE (Coordinación General del Comité Estatal de Planeación para el Desarrollo de Oaxaca) (2016). Diagnóstico de la cadena de valor mezcal en las regiones de Oaxaca. Oaxaca: COPLADE. Disponible en http://www.coplade.oaxaca.gob.mx/wp-content/ uploads/2017/04/Perfiles/AnexosPerfiles/6.\%20 CV\%20MEZCAL.pdf

Courlet, Claude y Bernard Pecqueur (1992). "Les systèmes industriels localisés en France: un nouveau modèle de développement". En Georges Benko y Alain Lipietz (eds.), Les Régions qui Gagnent. Districts et Réseaux: Les Nouveaux Paradigmes de la Géographie Économique. París: Presses Uni-versitaires, pp. 81-102.

De Grammont, Hubert C. (2004). "La nueva ruralidad en América Latina". En Revista Mexicana de Sociología, 66(1), 279-300. Disponible en http://mexicanadesociologia.unam.mx/docs/vol66/numesp/v66neal7.pdf

Duhart, Frédéric (2011). "Reflexiones desde la eco-antropología sobre el terroir". En Mundo agrario. Revista de estudios rurales, l1(22), primer semestre.

Flores López, María de Lourdes, Ever Sánchez Osorio, Ariel Vázquez Elorza y Joaline Pardo Núñez (2018). "Las tensiones en la denominación de origen del mezcal en Oaxaca, México". En Tlamati, 9(2), 32-36. Disponible en http://tlamati.uagro.mx/t92/t925.pdf

Goguitchaichvili, Avto, Miguel Cervantes Solano, Jesús Carlos Lazcano Arce, Mari Carmen Serra Puche, Juán Morales, Ana María Soler y Jaime Urrutia-Fucugauchi (2018). "Archaeomagnetic evidence of preHispanic origin of Mezcal". En Journal of Archaeological Science: Reports, 21, 504-511.

Hernández Moreno, María del Carmen y Angélica Villaseñor Medina (2014). "La calidad en el sistema agroalimentario globalizado". En Revista Mexicana de Sociología, 76(4), 557-582.

Iwadare, Miguel Ángel (2015). "La ruta del mezcal. Fábricas mezcaleras del altiplano potosino". En José de Jesús Hernández López y Miguel Ángel Iwadare (coords.). En torno a las bebidas alcohólicas mexicanas. Po- 
der, prácticas culturales y configuraciones regionales. Guadalajara: Centro Universitario de Los Altos-Universidad de Guadalajara.

Latour, Bruno (2001). La esperanza de Pandora: ensayos sobre la realidad de los estudios de la ciencia. Barcelona: Gedisa.

Linck, Thierry, Esteban Barragán López y François Casabianca (2006). "De la propiedad intelectual a la calificación de los territorios: lo que cuentan los quesos tradicionales". En Agroalimentaria 11(22), 99-109.

Lozano Armendares, Teresa (2015). "De cómo el chinguirito y el mezcal enfrentaron al aguardiente de Castilla, durante la Colonia”. En José de Jesús Hernández López y Miguel Ángel Iwadare (coords.). En torno a las bebidas alcohólicas mexicanas. Poder, prácticas culturales y configuraciones regionales. Guadalajara: Centro Universitario de Los Altos-Universidad de Guadalajara.

Merton, Robert (1946). "The focused interview and focus groups: Continuities and discontinuities". En Public Opinion Vuarterly, 5l(6), 550-566.

Merton, Robert, Marjorie Fiske y Patricia Kendall (1990). The focused interview: A manual of problems and procedures. Glencoe, IL: Free Press.

Muchnik, José y Denis Sautier (1998). Systèmes AgroAlimentaires Localisés et Construction de Territoires. Proposition d'Action Thématique Programmée. París: CIRAD.

Muchnik, José (2006). "Identidad territorial y calidad de los alimentos: procesos de calificación y competencias de los consumidores". En Agroalimentaria, 12(22), 89-98.

Plascencia de la Torre, María Fernanda y Lilian María Peralta Gordon (2018). "Análisis histórico de los mezcales y su situación actual desde una perspectiva ecomarxista”. En Eutopía: Revista de Desarrollo Económico Territorial, 14, 23-42.
Renard, Marie-Christine y David Rodolfo Domínguez Arista (2019). "The geographical indication of mezcal in Mexico: A tool of exclusion for small producers". En Alessandro Bonanno, Kae Sekine y Hart N. Feuer (eds.), Geographical Indication and Global Agri-Food. Londres: Routledge, pp. 173-185.

Requier-Desjardins, Denis (1997). Globalización y evolución de los sistemas de producción: La agroindustria rural y los "sistemas agroalimentarios localizados" en los países andinos. París: Université de Versailles.

Robles, Bernardo (2011). "La entrevista en profundidad: una técnica útil dentro del campo antropofísico". En Cuicuilco, 18(52), 39-49.

Sánchez Silva, Carlos (1999). "El comercio indígena en Oaxaca entre colonia y república”. En América Latina en la Historia Económica, 6(12).

Taylor, S. J. y R. Bogdan (1987). Introducción a los métodos cualitativos de investigación, t. 1. Barcelona: Paidós.

Valenzuela Zapata, Ana G., Aristarco Regalado Pinedo y Michiko Mizoguchi (2008). "Influencia Asiática en la Producción de Mezcal en la Costa de Jalisco. El caso de la Raicilla”. En México y la Cuenca del Pacífico (33), 91-116.

Villegas de Gante, Abraham y Fernando Cervantes Escoto (2011). "La genuinidad y tipicidad en la revalorización de los quesos artesanales mexicanos". En Estudios Sociales, 19(38), 145-164.

Walton, M. K. (1977). "The Evolution and Localization of Mezcal and Tequila in Mexico". En Revista Geográfica del Instituto Pan-americano de Geografía e Historia, 85, 113-132.

Zizumbo Villarreal, Daniel y Patricia Colunga-García Marín (2008). "Early coconut distillation and the origins of mezcal and tequila spirits in west-central Mexico". En Genetic Resources and Crop Evolution, 55(4), 493-510. 